\title{
Capsule Commentary on Bassett et al., Gender, Race, and Variations in the Evaluation of Microscopic Hematuria Among Medicare Beneficiaries
}

\author{
Yair Lotan, M.D. \\ J Gen Intern Med 30(4):491 \\ DOI: $10.1007 / \mathrm{s} 11606-014-3137-\mathrm{x}$ \\ (c) Society of General Internal Medicine 2014
}

UT Southwestern Medical Center at Dallas Department of Urology, Dallas, Texas, USA.
$\mathrm{W}$ hy do we evaluate patients with asymptomatic microscopic hematuria $(\mathrm{AMH})$ ? The primary concern is that these patients may have cancer (urothelial, renal, prostate), urolithiasis, medical renal disease or infections. Studies have found potentially lethal conditions in up to $10 \%$ of patients with microscopic hematuria. ${ }^{1}$ The AUA guidelines are very strict in their recommendations. "All patients 35 years or older should undergo cystoscopy, and upper urinary tract imaging is indicated in all adults with AMH in the absence of known benign causation." The premise is that this will lead to the least chance of missing malignancy. One problem for clinicians is that prevalence of AMH is common, ranging from 9 to $14 \%$, requiring many evaluations if the guidelines are followed strictly. Basset et al. studied 9,211 Medicare beneficiaries with incidental hematuria and found that hematuria evaluations were completed in only $14 \%{ }^{3}$ Women were significantly less likely to undergo a complete evaluation compared to men (10 vs. $22 \%$ ) and $69 \%$ of women failed to undergo any evaluation. Overall, only $23 \%$ of subjects were referred to urologists, who performed $98 \%$ of all procedures. Among 403 malignancies identified (4\% of participants), $81 \%$ were the result of complete evaluations.

A major concern for low rates of evaluation is the potential delay in diagnosis of cancer. Bladder cancer is diagnosed with muscle invasion in $25 \%$ of cases and half of those patients already have metastatic disease. Evaluation of patients in a timely fashion could theoretically improve detection of disease at an earlier stage. There have been several studies that have developed predictive models using clinical factors alone or in conjunction with urine-based tumor markers to improve detection of bladder cancer in patients with hematuria. ${ }^{4,5}$ These types of models can identify most patients with cancer while significantly reducing the number of evaluations in patients with $\mathrm{AMH}$, but there is always a risk that some cancers will be missed. Regardless, the current situation where the vast majority of hematuria patients undergo little to no evaluation is clearly not optimal. Risk stratification could potentially identify patients at highest risk to improve the odds that they will undergo appropriate evaluation.

Conflict of Interest: The author declares that he does not have a conflict of interest.

Corresponding Author: Yair Lotan, M.D.; UT Southwestern Medical Center at Dallas Department of Urology, 5323 Harry Hines Blvd. J8.122, Dallas, Texas 75390-9110, USA (e-mail: yair.lotan@utsouthwestern.edu).

\section{REFERENCES}

1. Khadra MH, Pickard RS, Charlton M, Powell PH, Neal DE. A prospective analysis of 1,930 patients with hematuria to evaluate current diagnostic practice. J Urol. 2000163 (2):524-7

2. Davis R, Jones JS, Barocas DA, Castle EP, Lang EK, Leveillee RJ, Messing EM, Miller SD, Peterson AC, Turk TM, Weitzel W; American Urological Association. Diagnosis, evaluation and follow-up of asymptomatic microhematuria (AMH) in adults: AUA guideline. J Urol. 2012188 (6 Suppl):2473-81. doi:10.1016/j.juro.2012.09.078. Epub 2012 Oct 24.

3. Bassett J, Alvarez J, Koyama T, Resnick M, You C, Ni S, Penson D, Barocas D. Gender, Race, and Variations in the Evaluation of Microscopic Hematuria Among Medicare Beneficiaries. JGIM. doi:10.1007/s11606-0143116-2.

4. Loo RK, Lieberman SF, Slezak JM, Landa HM, Mariani AJ, Nicolaisen G, Aspera AM, Jacobsen SJ. Stratifying risk of urinary tract malignant tumors in patients with asymptomatic microscopic hematuria. Mayo Clin Proc. 201388 (2):129-38. doi:10.1016/j.mayocp.2012.10.004. Epub 2013 Jan 9. PMID:23312369

5. Lotan Y, Svatek RS, Krabbe LM, Xylinas E, Klatte T, Shariat SF. Prospective external validation of a bladder cancer detection model. J Urol. 2014192 (5):1343-8. doi:10.1016/j.juro.2014.05.087. Epub 2014 May 21.PMID:24859442 